\title{
Modelo Multiobjetivo para Seleção de Portfólios com Restrição de Cardinalidade, Custo de Transação e Valor em Risco Condicional
}

\author{
G.P. HANAOKA ${ }^{1 *}$, R.T.N. CARDOSO ${ }^{1}$ e F.D. PAIVA ${ }^{2}$
}

Recebido em 13 abril, 2016 / Aceito em 6 novembro, 2016

\begin{abstract}
RESUMO. Este trabalho apresenta um modelo multiobjetivo para seleção de portfólios de ações do mercado financeiro, que leva em consideração a restrição de cardinalidade, os custos de transação e os limites de investimento para cada ativo e para grupos de ativos. As funções-objetivo consideram o valor em risco condicional (CVAR - Conditional Value-at-Risk) como medida de risco e o valor esperado dos retornos históricos ponderados pelas proporções de investimento, descontados os custos de transação. Para a otimização do modelo foi utilizado um algoritmo genético multiobjetivo. Resultados mostram a capacidade do algoritmo em encontrar várias soluções eficientes, bem como a capacidade do modelo em auxiliar a tomada de decisão na escolha de portfólios que apresentem uma boa relação entre risco e retorno, para uma dada cardinalidade.
\end{abstract}

Palavras-chave: otimização multiobjetivo, seleção de portfólios, valor em risco condicional, algoritmos genéticos.

\section{INTRODUÇÃO}

O estudo da formação de portfólios ótimos com ativos do mercado financeiro é largamente estudado na área de otimização aplicada a finanças. O precursor deste campo investigativo foi Harry Markowitz [11], que propôs um modelo de média-variância buscando a diversificação do investimento para melhorar a relação risco $\times$ retorno, utilizando como medida de risco a covariância entre os retornos dos ativos e como medida de retorno esperado a média dos retornos dos ativos, ambos ponderados pelas proporções de investimentos em cada ativo. Este modelo consiste em um problema de programação quadrática, sendo facilmente resolvido utilizando métodos determinísticos.

Trabalhos posteriores construíram modelos utilizando as ideias de Markowitz, porém indicando melhorias para o modelo através da adição de restrições como cardinalidade, lote mínimo de

\footnotetext{
*Autor correspondente: Gustavo Peixoto Hanaoka.

${ }^{1}$ Programa de Pós-Graduação em Modelagem Matemática e Computacional, PPGMMC-CEFET-MG, Centro Federal de Educação Tecnológica de Minas Gerais, 30510-000 Belo Horizonte, MG, Brasil. E-mails: gustavopph@ gmail.com; rodrigoc@des.cefetmg.br

${ }^{2}$ Programa de Pós-Graduação em Administração, PPGA-CEFET-MG, Centro Federal de Educação Tecnológica de Minas Gerais, 30510-000 Belo Horizonte, MG, Brasil. E-mail: fpaiva@ dcsa.cefetmg.br
} 
transação e setores de capitalização [6], [17]. Além da incorporação de restrições no modelo de Markowitz, trabalhos posteriores também fizeram uma abordagem multiobjetivo explícita, discriminando as funções de risco e retorno [7], bem como adicionando uma outra função objetivo para restringir a cardinalidade dos portfólios [2].

Contudo, um outro debate que surge tem a ver com o problema de se considerar a variância como medida de risco, pois os valores positivos de retorno são levados em consideração da mesma forma que os valores negativos de retorno. Para tanto, propõem-se tratar o risco de um portfólio pela ótica das perdas, como é o caso das medidas de downside risk. Uma forma de risco que se enquadra nessa classificação é o valor em risco condicional (CVaR - Conditional Value at Risk) [13], definido a partir do valor em risco (VaR - Value at Risk). Dentre os trabalhos que utilizam o VaR e CVaR pode-se citar aqueles que apresentam a formulação de modelos que os utilizam como função objetivo ou como restrição em modelos de maximização do retorno [1], [14]. Trabalhos propondo um modelo multiobjetivo utilizando o CVaR como medida de risco podem ser encontrados em [15] e [16].

Deve-se levar em consideração que, em geral, a adição de restrições faz com que a complexidade do problema aumente significativamente. Um exemplo é a inclusão da restrição de cardinalidade, que faz com que seja necessário escolher a carteira com uma quantidade de ativos estipulada, a partir do conjunto total de ativos disponíveis para investimento, o que torna o problema da seleção de portfólios um problema NP-Difícil [9]. Este fato faz com que seja justificável a utilização de metaheurísticas para a resolução do problema.

Recentemente, o trabalho [18] propôs um modelo de otimização baseado no modelo de Markowitz levando em conta os custos de transação, restrição de cardinalidade, limites de investimentos em ativos e em grupos de ativos, considerando como risco a variância. O modelo proposto utilizou uma única função objetivo ponderando os valores de risco e retorno (método ponderado [8]), resolvido por meio de um algoritmo memético mono-objetivo. No mesmo trabalho [18], foi feito um estudo a respeito do impacto da utilização de diversos valores de custos de transação e da consideração de presença e ausência das restrições.

O presente trabalho apresenta um modelo de otimização multiobjetivo com restrição de cardinalidade, custos de transação e risco modelado pelo CVaR, que considera apenas o risco pelas perdas esperadas. Para a otimização do modelo foi proposto um algoritmo genético com operadores apropriados para o problema. A opção por um modelo multiobjetivo decorreu do fato de que o problema na forma ponderada apresenta algumas desvantagens, como a necessidade de escolha de fatores de ponderação capazes de gerar soluções com boa cobertura no espaço de objetivos [8]. Além disso, a resolução de problemas multiobjetivos por meio de algoritmos genéticos busca encontrar ao fim de cada execução um conjunto de soluções não dominadas ao invés de uma única solução a cada execução.

O trabalho está organizado como segue: a Seção 2 apresenta o modelo utilizado neste trabalho. A Seção 3 descreve o algoritmo proposto para a otimização do modelo. A Seção 4 descreve as entradas utilizadas no modelo, os parâmetros do algoritmo e as medidas de desempenho utiliza- 
das na avaliação das soluções. A Seção 5 apresenta os resultados obtidos e, por fim, na Seção 6 faz-se as conclusões a respeito do trabalho realizado.

\section{MODELO DE COMPOSIÇÃO DE PORTFÓLIO}

O presente trabalho utilizou-se para a composição de portfólios de um modelo de otimização multiobjetivo, considerando: o CVaR como medida de risco, o retorno como descrito em [11], os custos de transação como em [18], e outras restrições com o intuito de aproximar o modelo utilizado a comportamentos reais no mercado financeiro.

De acordo com [10] e [14], tomando uma função de perda $f(\mathbf{w}, \mathbf{r})$ associada ao vetor $\mathbf{w} \subset \mathbb{R}^{N}$ correspondente as proporções de investimentos e um vetor aleatório de parâmetros do mercado que resultam em $J$ cenários de retorno $\mathbf{r}^{J} \in \mathbb{R}^{J \mathbf{x} N}$ com probabilidade de ocorrência $\pi_{j}$, a perda $f(\mathbf{w}, \mathbf{r})$ tem uma distribuição em $\mathbb{R}$ induzida por $\mathbf{r}$, que pode ser chamada de $p(\mathbf{r})$. A probabilidade dessa perda não exceder um valor $\alpha$ é dada pela equação (2.1).

$$
\Psi(\mathbf{w}, \alpha)=\int_{f(\mathbf{w}, \mathbf{r}) \leq \alpha} p(\mathbf{r}) d r
$$

$\mathrm{O}$ valor em risco $\beta$-VaR para uma variável aleatória representa a perda relacionada com w. Considerando um nível de confiança $\beta \in(0,1)$, o $\beta$-VaR é dado pela equação (2.2).

$$
\zeta_{\beta}(\mathbf{w}, \alpha)=\min \{\zeta \in \mathbb{R}: \Psi(\mathbf{w}, \zeta) \geq \alpha\}
$$

Uma vez que o VaR não mensura as perdas que o ultrapassam, considera-se o valor em risco condicional $\beta$-CVaR como o valor esperado das perdas que ultrapassam o $\beta$-VaR, descrito pela equação (2.3).

$$
\phi_{\beta}(\mathbf{w}, \alpha)=(1-\alpha)^{-1} \int_{f(\mathbf{w}, r) \geq \zeta_{\beta}(\mathbf{w})} f(\mathbf{w}, \mathbf{r}) p(\mathbf{r}) d r .
$$

Considerando o caso discreto em que a função de perda é dada pela equação (2.4), pode-se trabalhar com a forma discreta do $\beta$-CVaR descrita por $\tilde{F}_{\beta}(\mathbf{w}, \zeta)$ na equação (2.5).

$$
\begin{gathered}
f(\mathbf{w}, \mathbf{r})=-\left[w_{1} r_{1}+\cdots+w_{n} r_{n}\right]=-\mathbf{w}^{T} \mathbf{r} \\
\tilde{F}_{\beta}(\mathbf{w}, \zeta)=\zeta+(1-\beta)^{-1} \sum_{j=1}^{J} \pi_{j}\left[f\left(w, r_{j}\right)-\zeta\right]^{+}
\end{gathered}
$$

Neste caso, $\zeta$ é o VaR correspondente e $\pi_{j}$ é a probabilidade de ocorrência do cenário $j$.

Matematicamente, uma medida do retorno esperado pode ser considerada como os valores passados de retorno $\mathbf{r}_{j}$ ponderados pelas proporções de investimento $\mathbf{w}$ descrita pela equação (2.6).

$$
\mathbb{E}(\mathbf{r}, \mathbf{w})=\mathbf{w}^{T} \overline{\mathbf{r}}
$$

Como no cenário real existem os custos de transações que impactam no retorno do portfólio, o retorno esperado pode ser descrito pela equação (2.7) como feito em [18], em que considera-se o 
custo de transação linear em partes, sendo $\mathbf{T}_{c}$ o vetor correspondente ao custo de transação gasto ao investir em cada ativo.

$$
\mathbb{E}(\mathbf{r}, \mathbf{w})=\mathbf{w}^{T} \overline{\mathbf{r}}-\mathbf{T}_{c}^{T} \mathbf{w}
$$

O modelo estudado neste trabalho, descrito pelas equações (2.8) - (2.10d), consiste em um modelo multiobjetivo restrito em que se busca maximizar o retorno e minimizar o risco, respeitando as restrições.

$$
\begin{gathered}
\min _{w_{1}, \ldots, w_{N}} \quad \zeta+(1-\alpha)^{-1} \sum_{j=1}^{J} \pi_{j}\left[f\left(w, r_{j}\right)-\zeta\right]^{+} \\
\min _{w_{1}, \ldots, w_{N}}-\sum_{i=1}^{N} w_{i} \mu_{i}+\mathbf{T}_{c} w \\
\text { s.a. }\left\{\begin{array}{l}
\mathbf{w} \cdot \mathbf{1}+\mathbf{T}_{c} \mathbf{w}=1 \\
\mathbf{l}^{z} \leq \mathbf{w}^{z} \leq \mathbf{u}^{z}, \mathbf{l} \geq \mathbf{0}, \mathbf{u} \geq \mathbf{0} \\
\mathbf{L s} \leq \mathbf{A}^{z} \cdot \mathbf{w}^{z} \leq \mathbf{U s}, \mathbf{L s} \geq \mathbf{0}, \mathbf{U s} \geq \mathbf{0} \\
\mathbf{z}^{T} \cdot \mathbf{1}=k
\end{array}\right.
\end{gathered}
$$

As funções objetivo de minimização do risco e maximização do retorno são descritas respectivamente pelas equações (2.8) e (2.9) em que $\mu_{i}$ corresponde a média dos retornos de cada ativo $i\left(\mu_{i}=\bar{r}_{i}\right)$. A restrição da equação (2.10a) garante que o somatório das proporções de investimento com os custos de transação exigidos é igual ao total do capital disponível; a restrição da equação (2.10b) garante que as proporções de investimento em cada ativo seja limitada inferior (l) e superiormente (u); a restrição da equação (2.10c) limita inferior (Ls) e superiormente (Us) os grupos (ou setores) de ativos, sendo os grupos determinados na matriz $A$; a restrição da equação (2.10d) representa a restrição de cardinalidade, em que se limita o número de ativos no portfólio, podendo este conter menos ativos do que a quantidade total de ativos disponíveis para investimento. A variável de controle $\mathbf{z}$ consiste em um vetor que possui o mesmo número de elementos que a quantidade de ativos totais, sendo que os ativos presentes no portfólio possuem o valor "1" na posição correspondente do vetor $\mathbf{z}$, enquanto os ativos não presentes no portfólio possuem o valor "0" na posição correspondente do vetor $\mathbf{z}$.

\section{O ALGORITMO PROPOSTO}

O modelo proposto vale-se de um algoritmo genético multiobjetivo com operadores específicos para cruzamento e mutação baseados em [12] e [18], além dos operadores de seleção e arquivamento baseados no algoritmo SPEA2 (Strength Pareto Evolutionary Algorithm 2) [20].

A codificação das soluções foi feita em uma matriz, na qual cada linha representava um portfólio, sendo este um indivíduo. Dessa forma, os ativos presentes no portfólio possuíram em sua posição, no vetor, um valor real representando a proporção de investimento nesses ativos. Os valores de risco e retorno foram guardados separadamente. 
Para a geração da população inicial foram determinados aleatoriamente $k$ ativos e suas correspondentes proporções de investimentos, respeitando as restrições de limites do modelo.

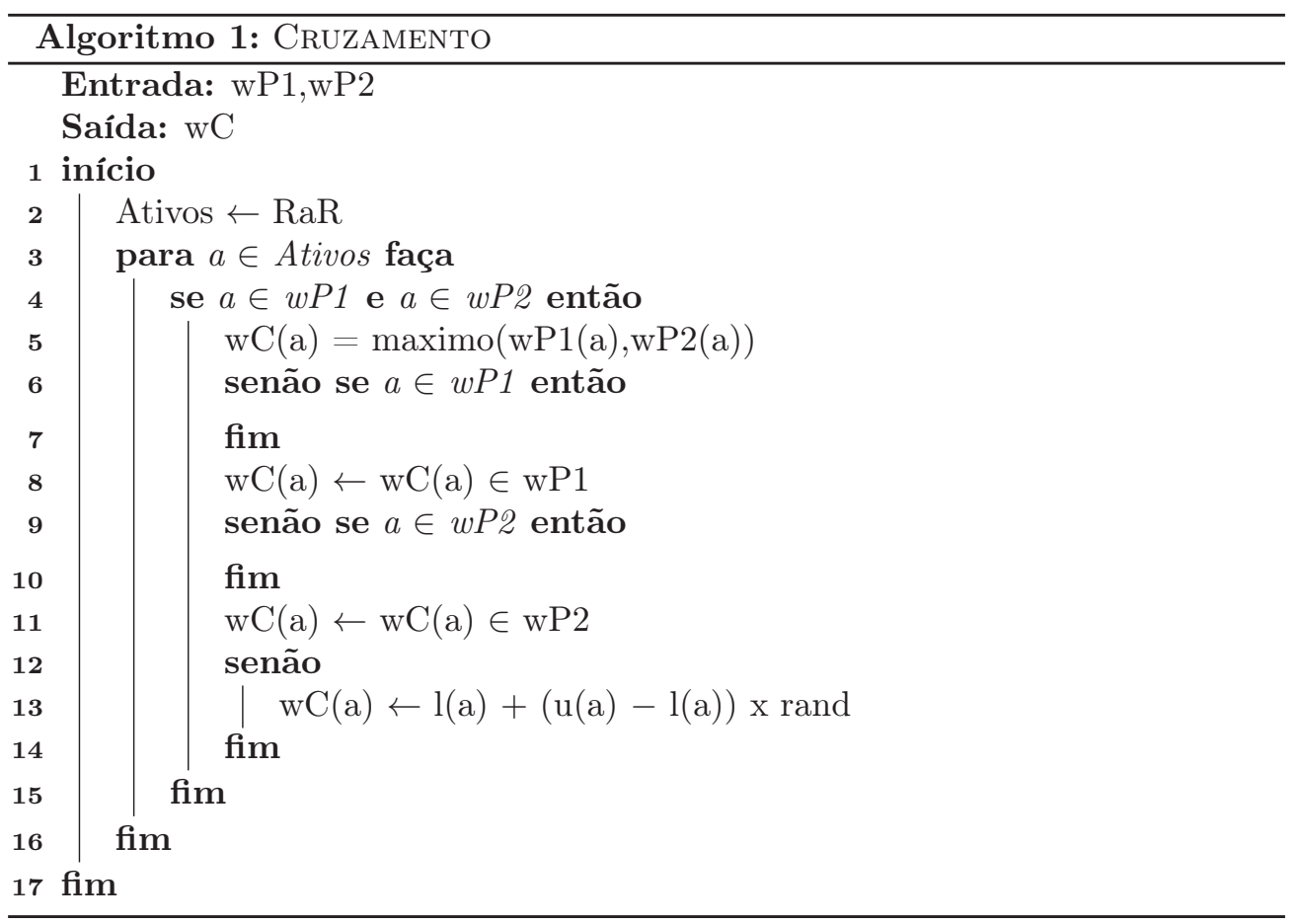

O cruzamento realizado pelo algoritmo utiliza dois indivíduos da população corrente para gerar dois novos indivíduos. Os indivíduos gerados podem herdar ativos ou proporções de investimentos dos indivíduos geradores, bem como obter novos ativos e proporções de investimentos não presentes nos indivíduos geradores. Dessa forma, aplicou-se o operador de cruzamento RAR (Random Assorting Recombination), introduzido em [12], para selecionar quais ativos o indivíduo gerado iria possuir e logo após fez-se a determinação das proporções de investimento de cada ativo, conforme mostrado no Algoritmo 1. Este processo pode ocorrer de três maneiras distintas: (I) se o ativo está presente em ambos os parentes, o indivíduo herda a maior proporção de investimento entre os dois parentes para o ativo em questão; (II) se o ativo está presente em apenas um parente o indivíduo herda a proporção correspondente a este ativo; (III) se o ativo não está presente em nenhum parente, é gerada aleatoriamente uma proporção de investimento, respeitando os limites inferiores e superiores deste valor, bem como o custo de transação. No algoritmo citado, $w P 1$ e $w P 2$ são os portfólios dos indivíduos da população selecionados para o cruzamento, $w C$ é o portfólio do indivíduo a ser gerado, $l$ e $u$ se referem aos limites inferiores e superiores de cada ativo e rand consiste na geração de um número aleatório uniformemente distribuído no intervalo $[0,1]$. 
Após a geração de novos indivíduos a partir do operador de cruzamento, faz-se a mutação dos novos indivíduos gerados de acordo com um valor de probabilidade, a fim de inserir ainda mais diversidade a população.

São três os tipos de mutação possíveis: (I) permutação de proporções de investimentos entre os ativos; (II) geração de uma nova proporção de investimento para o ativo em questão; (III) substituição de ativos presentes no portfólio por ativos não presentes, caso a cardinalidade em questão seja menor que o número de ativos. O Algoritmo 2 descreve o operador de mutação proposto, sendo $w C$ o portfólio do indivíduo que irá mutar, $N$ a quantidade total de ativos, $k$ a cardinalidade, $n C$ a quantidade de ativos que serão trocados na mutação (caso (III)), $l$ e $u$ os limites inferiores e superiores de cada ativo, rand um número aleatório uniformemente distribuído no intervalo $[0,1]$ e randi( $\operatorname{Lim})$ um número inteiro aleatório com limite superior igual a Lim.

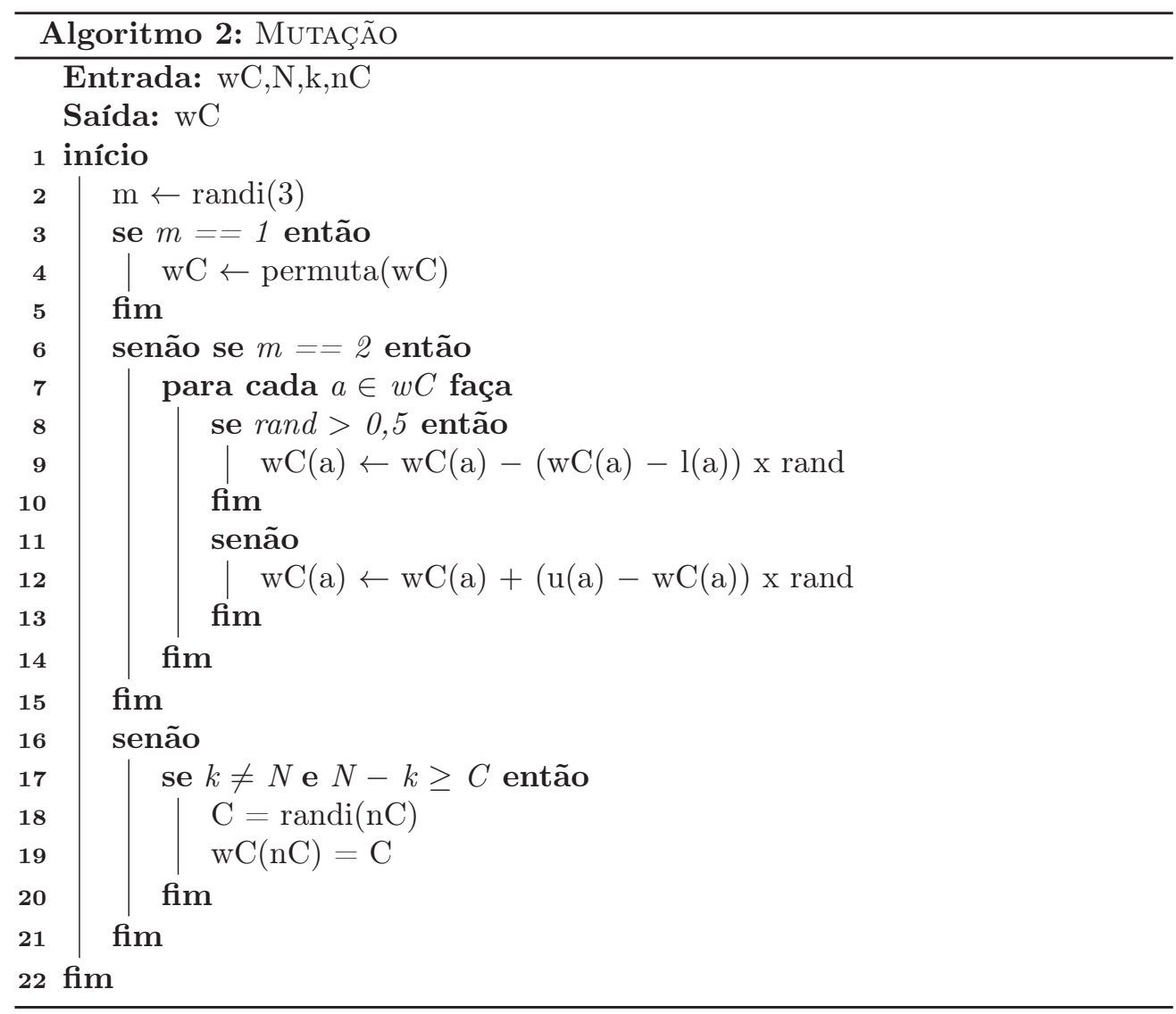

Foram testados empiricamente os métodos de seleção e arquivamento utilizados em algoritmos multiobjetivo e decidiu-se por utilizar as técnicas propostas no algoritmo Strength Pareto Evolutionary Algorithm 2 (SPEA2) [20], dada a vasta quantidade de trabalhos encontrados na 
literatura comprovando as vantagens deste método. No algoritmo SPEA2 é utilizado um conjunto de possíveis soluções do problema (população) e um conjunto auxiliar (arquivo). A população é gerada aleatoriamente e o arquivo é tomado como vazio. Após a aplicação dos operadores de cruzamento e mutação, o valor de fitness de cada indivíduo é calculado baseado na quantidade de indivíduos que este indivíduo domina, juntamente com uma medida de densidade, utilizando uma técnica de clusterização. Os indivíduos não dominados são arquivados e a seleção é realizada entre os indivíduos do arquivo. O conjunto das melhores soluções encontradas pelo algoritmo consiste nas soluções do arquivo.

\section{SELEÇÃO DOS DADOS, MEDIDAS DE DESEMPENHO, PARÂMETROS DO MODELO E ALGORITMO}

O conjunto de ações utilizadas fizeram parte do portfólio teórico do índice Bovespa (IBOVESPA) [4] no terceiro trimestre de 2015. O IBOVESPA é um indicador de desempenho médio das cotações dos ativos de maior negociabilidade da BMF\&Bovespa, bolsa de valores brasileira. No total haviam 63 ações, porém aquelas que não apresentaram cotações em $95 \%$ dos dias do período de 1/10/2013 a 30/12/2013 foram descartadas, restando 55 ações. Cada ativo foi identificado por um número natural entre um (1) e cinquenta e cinco (55), que facilitou a manipulação dos dados e resultados, conforme mostrados na Tabela 1.

Tabela 1: Ativos utilizados.

\begin{tabular}{|l|l|l|l|l|}
\hline (1) ABEV3 & (12) CIEL3 & (23) EQTL3 & (34) LAME4 & (45) RENT3 \\
\hline (2) BBAS3 & (13) CMIG4 & (24) FIBR3 & (35) LREN3 & (46) SANB11 \\
\hline (3) BBDC3 & (14) CPFE3 & (25) GGBR4 & (36) MRFG3 & (47) SBSP3 \\
\hline (4) BBDC4 & (15) CPLE6 & (26) GOAU4 & (37) MRVE3 & (48) SUZB5 \\
\hline (5) BRAP4 & (16) CSAN3 & (27) GOLL4 & (38) MULT3 & (49) TBLE3 \\
\hline (6) BRFS3 & (17) CSNA3 & (28) HGTX3 & (39) NATU3 & (50) TIMP3 \\
\hline (7) BRKM5 & (18) CTIP3 & (29) HYPE3 & (40) OIBR3 & (51) UGPA3 \\
\hline (8) BRML3 & (19) CYRE3 & (30) ITSA4 & (41) PCAR4 & (52) USIM5 \\
\hline (9) BVMF3 & (20) ELET3 & (31) ITUB4 & (42) PETR3 & (53) VALE3 \\
\hline (10) CCRO3 & (21) EMBR3 & (32) JBSS3 & (43) PETR4 & (54) VALE5 \\
\hline (11) CESP6 & (22) ENBR3 & (33) KROT3 & (44) RADL3 & (55) VIVT4 \\
\hline
\end{tabular}

Definidas as ações e o período dos dados, foram calculados os retornos baseados nos valores de fechamentos diários de negociação dos ativos financeiros da amostra no período utilizado, por meio da equação (4.1). A quantidade de dados utilizada como entrada totalizam 60 retornos diários de cada ativo. Os dados econômicos foram extraídos no terminal da Bloomberg L.P. [3].

$$
r(t)=\ln \left(\frac{\text { Fechamento }(t)}{\operatorname{Fechamento}(t-1)}\right)
$$


O algoritmo foi executado 30 vezes para cada cardinalidade e foi considerado um conjunto final de soluções unindo as soluções não dominadas de todas as execuções. Os parâmetros utilizados no algoritmo são listados na Tabela 2.

Tabela 2: Parâmetros do algoritmo.

\begin{tabular}{|l|c|}
\hline \multicolumn{1}{|c|}{ Parâmetros } & Valor \\
\hline Número de Indivíduos & 50 \\
\hline Número de Gerações & 1000 \\
\hline Tamanho do Arquivo & 50 \\
\hline Probabilidade de Cruzamento & 1 \\
\hline Paramâmetro do Cruzamento $c$ (RAR) & 1 \\
\hline Probabilidade de Mutação & 0,05 \\
\hline Paramâmetro da Mutação $n C$ & 2 \\
\hline
\end{tabular}

O limite inferior de proporção de investimento em cada ativo no portfólio foi mantido fixo igual a $l=0,01$, enquanto o limite superior foi mantido igual a $u=0,99$. Foram considerados cinco setores de investimentos compostos por todos os ativos do portfólio, uma vez que decidiu-se não classificar os ativos em grupos. O custo de transação foi mantido fixo em $T_{c}=0,003=30 \mathrm{bps}{ }^{1}$ para todos os ativos, valor este que condiz com as práticas do mercado brasileiro. O nível de confiança utilizado pela medida de risco foi estipulado como $\beta=0,9$.

As avaliação do modelo e do algoritmo foi feita através da comparação entre soluções encontradas para cada uma das cardinalidades $k=5,10,15,20,30$. As medidas de desempenho utilizadas neste trabalho consistem em: (I) número de soluções não dominadas ao final das execuções do algoritmo; (II) número de portfólios diferentes nas fronteiras não dominadas; (III) máximo valor de Índice de Desempenho do Portfólio (IDP) encontrado; (IV) hipervolume (ou S-Metric) dos conjuntos de soluções. A medida (I) reflete a capacidade do algoritmo de encontrar soluções, quanto mais melhor. A medida (II) conta quantos portfólios diferentes foram encontrados, não em relação a proporção de investimentos e sim em relação aos ativos escolhidos, buscando explorar o espaço de busca (exploitation). A medida (III), descrita na equação (4.2), busca valorizar as soluções com o maior valor excedente de retorno em relação ao risco, ou seja, quanto maior o valor de retorno e menor o valor de risco, maior será o valor da medida IDP. Para um mesmo valor de risco, a solução com maior valor de retorno se sobressai, enquanto que para um mesmo valor de retorno, a solução com menor valor de risco se sobressai, em relação a medida IDP. A medida (IV) calcula a área no espaço de objetivos dominada pelo conjunto de soluções e limitada por um valor de referência [5], [19]. Este valor de referência, neste caso, é o par ordenado formado pelo pior valor de risco e pelo pior valor de retorno encontrados em relação a todas

${ }^{1} 1$ basis points $(\mathrm{bps})=0,01 \%=0,0001$ 
as soluções a serem comparadas. Quanto maior o valor de hipervolume, maior a convergência e diversidade do conjunto de soluções considerado (exploration).

$$
I D P=\frac{\sum_{i=1}^{N} w_{i} \mu_{i}-\mathbf{T}_{c} w}{\zeta+(1-\alpha)^{-1} \sum_{j=1}^{J} \pi_{j}\left[f\left(w, r_{j}\right)-\zeta\right]^{+}}
$$

\section{RESULTADOS}

A Figura 1 apresenta as fronteiras não dominadas encontradas para cada uma das cardinalidades. Observa-se que a fronteira obtida para $k=5$ foi aquela que obteve a maior convergência e diversidade, apresentando também soluções extremas melhores que as outras fronteiras. Utilizando-se a cardinalidade $k=10$ também obteve-se uma boa cobertura do espaço de objetivos, tendo algumas soluções de menor risco apresentando graficamente uma semelhança com as soluções de menor risco da fronteira para $k=5$. Para as demais cardinalidades, percebe-se uma baixa cobertura do espaço de objetivos, tendo o tamanho das fronteiras diminuído progressivamente conforme a cardinalidade foi aumentada. Além disso, observa-se que para $k=15$ e $k=20$ uma boa parte das soluções de suas fronteiras possuíram retornos negativos, tendo a cardinalidade $k=30$ apresentado apenas soluções com retornos negativos, o que as tornam ineficientes.

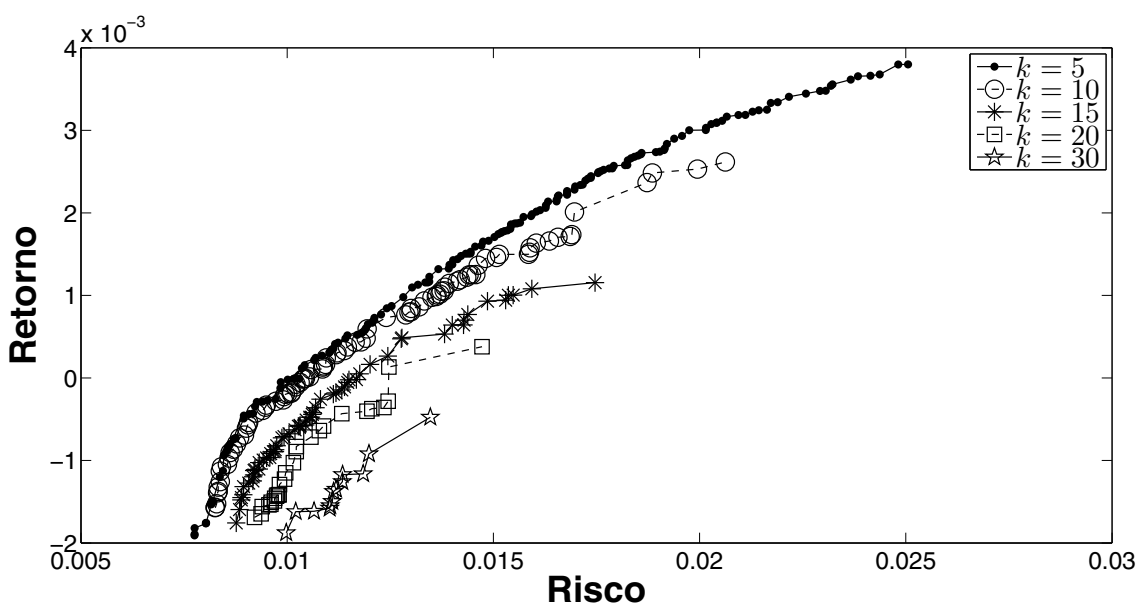

Figura 1: Fronteiras de soluções não dominadas para diferentes valores de cardinalidade $(k)$ : 5 , $10,15,20$ e 30.

Na Tabela 3 observa-se que o número de soluções encontradas diminui conforme a cardinalidade aumenta. A cardinalidade que apresentou o maior número de soluções foi $k=5$, seguida de $k=10$. No entanto, mesmo para cardinalidades maiores o algoritmo foi capaz de encontrar portfólios diferentes no sentido da escolha de diferentes ativos para compor os portfólios, sem levar em consideração a proporção de investimento em cada ativo. Em relação ao máximo valor de IDP de cada cardinalidade, pode-se afirmar que como as fronteiras de $k=5$ e $k=10$ encontraram soluções de maiores retornos, estas consequentemente possuíram melhores valores 
de IDP. Como para $k=30$ todos os retornos encontrados foram negativos, seu valor máximo encontrado de IDP foi também negativo. Os valores de hipervolume encontrados corroboram a representação gráfica das fronteiras, tendo sido $k=5$ e $k=10$ as cardinalidades que obtiveram as fronteiras de soluções mais diversificadas e que mais convergiram de todas as cardinalidades.

Tabela 3: Medidas de desempenho para diferentes valores de cardinalidade $(k): 5,10$, 15,20 e 30 .

\begin{tabular}{|l|c|c|c|c|c|}
\hline & $k=5$ & $k=10$ & $k=15$ & $k=20$ & $k=30$ \\
\hline No. Soluções & 165 & 90 & 60 & 28 & 13 \\
\hline No. Port. Diferentes & 17 & 32 & 29 & 20 & 12 \\
\hline Máx. IDP & 0,153683 & 0,131715 & 0,067802 & 0,025649 & $-0,035363$ \\
\hline Hipervolume & 0,653968 & 0,579215 & 0,425333 & 0,321885 & 0,191709 \\
\hline
\end{tabular}

A Figura 2 representa a distribuição do risco em relação a cada uma das cardinalidades. Tanto a cardinalidade $k=5$, como a cardinalidade $k=10$ apresentam uma grande extensão de risco possibilitando a escolha de soluções diversas.

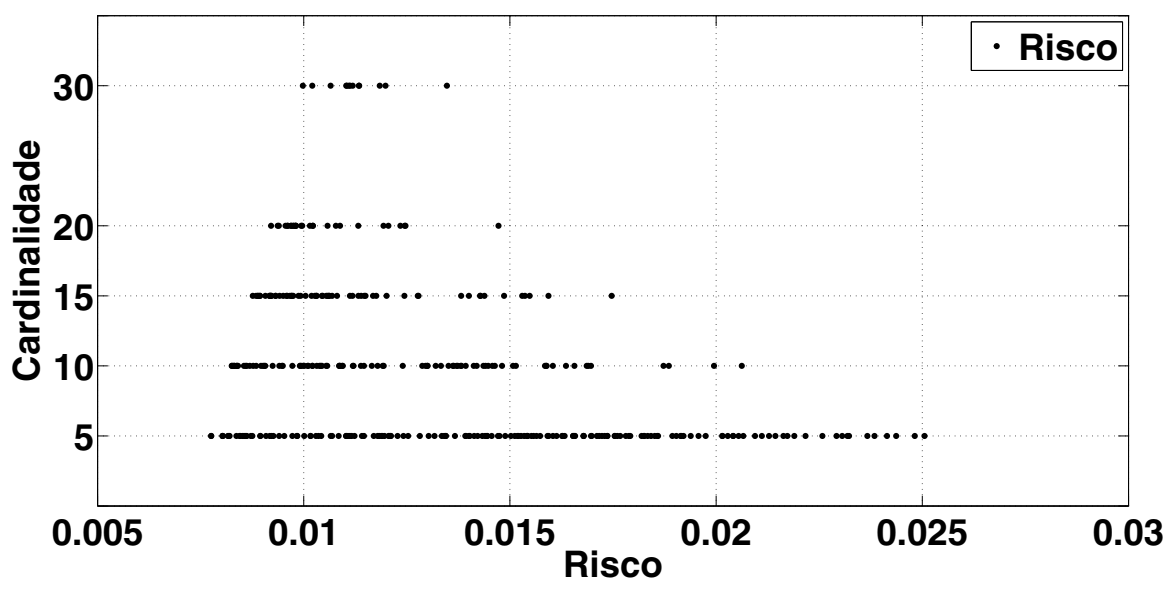

Figura 2: Risco $\times$ Cardinalidade

Já a Figura 3 representa a extensão do retorno obtido por cada cardinalidade. Fica claro que além de retornos extremos mais altos, as cardinalidae $k=5$ e $k=10$ obtiveram uma gama maior de retornos, inclusive compreendendo toda a extensão obtida pelas demais cardinalidades. Constata-se também que para as cardinalidades $k=15,20,30$ a maioria dos retornos obtidos pelas soluções são negativos, portanto, ineficientes.

Em uma última análise, vale apresentar a distribuição dos retornos dos ativos utilizados como entrada do modelo, Figura 4. Dentre os ativos utilizados, 34 dos 55 ativos apresentaram médias 


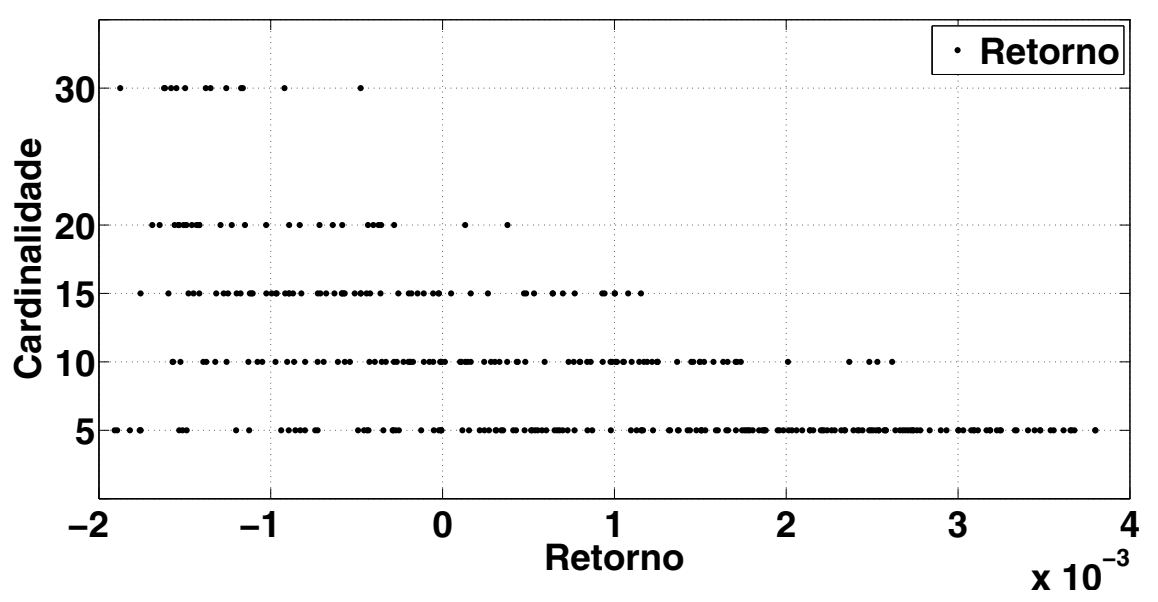

Figura 3: Retorno $\times$ Cardinalidade

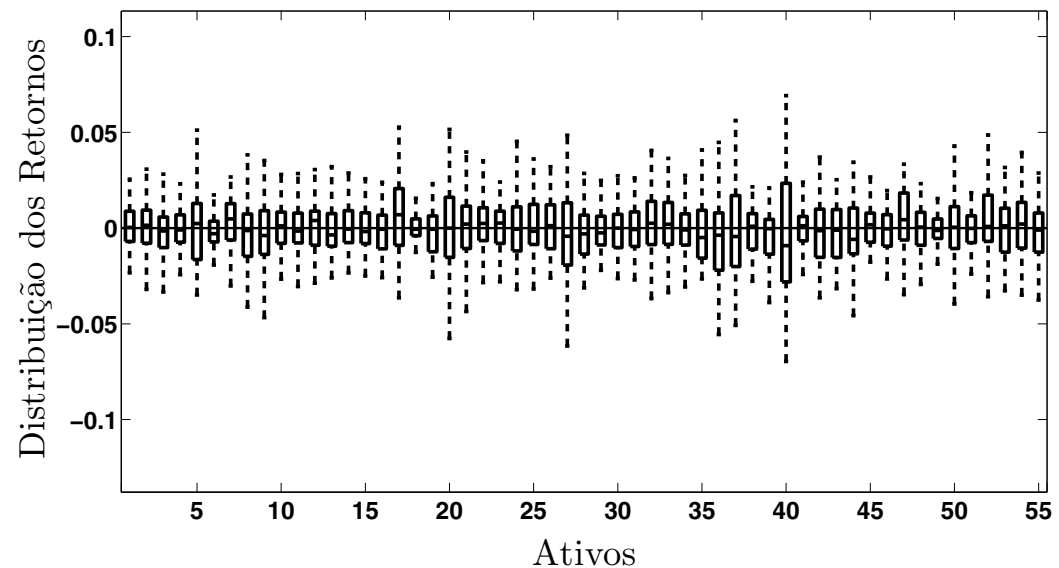

Figura 4: Distribuição dos retornos dos ativos no período estudado.

de retornos menores ou iguais a zero, sendo que 33 dos 55 ativos apresentaram mediana menor ou igual a zero. Esse fato pode sugerir uma possível justificativa para a ineficiência dos retornos das soluções obtidas pelas cardinalidades $k=15,20,30$, uma vez que aumentando a quantidade de ativos no portfólio, aumenta-se a chance de adicionar ao portfólio aqueles ativos que possuíam retornos abaixo ou iguais a zero. Vê-se então que o modelo otimizado pelo algoritmo proposto é capaz de encontrar soluções viáveis mesmo quando de posse de um conjunto de ativos com retornos em sua grande parte desfavoráveis para investimento.

Utilizando a metodologia proposta é necessário que se faça uma análise para a escolha da cardinalidade apropriada a ser adotada em uma situação real de investimento no mercado. Para este 
trabalho, as cardinalidades que resolveram o problema dessa escolha se limitaram aos portfólios encontrados com cardinalidades $k=5$ ou $k=10$, uma vez que além de apresentarem maiores opções de escolhas, estes ainda apresentaram soluções com melhores relações risco $\times$ retorno. Dessa forma, caberia ao decisor optar por escolher o portfólio dentre as fronteiras dessas cardinalidades de acordo com sua aversão ao risco e sua expectativa de retorno.

\title{
6 CONCLUSÕES
}

Neste trabalho utilizou-se um modelo multiobjetivo restrito para composição de portfólios levando em consideração os custos de transação, tendo como medida de risco o CVaR e como medida de retorno a média ponderada dos retornos históricos dos ativos, descontada dos custos de transação. O modelo foi otimizado via algoritmo genético com operadores específicos propostos para a resolução do problema. Constatou-se que o modelo foi capaz de gerar portfólios viáveis com restrição de cardinalidade, apresentando melhores resultados em relação às medidas de desempenho em geral para as menores cardinalidades, uma vez que grande parte dos ativos apresentaram retornos negativos ou iguais a zero no período estudado. No que tange a otimização pelo algoritmo proposto, pode-se concluir que os operadores propostos foram eficientes em relação a convergência e diversidade das soluções, apresentando diversidade tanto no espaço de busca, como de valores de risco e retorno no espaço de objetivos. Em trabalhos futuros pretende-se aperfeiçoar a convergência do algoritmo bem como buscar melhorias no modelo, aproximando-o cada vez mais de situações reais de compra e venda de ações no mercado financeiro.

\begin{abstract}
This work presents a multiobjective model for portfolio selection that takes into account cardinality constraint, transaction costs and investment limits for each asset and asset groups. The objective functions consider the conditional value-at-risk (CVaR) as the risk measure and the expected value of the historical returns weighted by the investment proportions, discounted the transactions costs. To cope with the model, a specific version of a multiobjective genetic algorithm is used. Results show the ability of the algorithm to achieve several efficient solutions, as well the usability of the proposed model to assist the market investor decision in obtaining potfolios with good relation between risk and return according to a given cardinality.
\end{abstract}

Keywords: multiobjective optimization, portfolio selection, conditional value at risk, genetic algorithms.

\section{REFERÊNCIAS}

[1] G.J. Alexander \& A.M. Baptista. A comparison of var and cvar constraints on portfolio selection with the mean-variance model. Management Science, 50(9) (2004), 1261-1273.

[2] K.P. Anagnostopoulos \& G. Mamanis. A portfolio optimization model with three objectives and discrete variables. Computers \& Operations Research, 37(7) (2010), 1285-1297. 
[3] L.P. Bloomberg. "Historical Stock Price for Ibovespa (data)", 1/10/13 a 30/12/13. Retirado em 15/12/15, Bloomberg Database.

[4] Índice Bovespa, Abril 2016, disponível em <http://www.bmfbovespa.com.br/pt_br/produtos/indices/ indices-amplos/indice-ibovespa-ibovespa-composicao-da-carteira.htm $>$. Acesso em: 1 de Abril de 2016.

[5] J. Branke. Consideration of partial user preferences in evolutionary multiobjective optimization, em "Multiobjective optimization", pp. 157-178, Springer Berlin, Heidelberg (2008).

[6] T-J Chang, N. Meade, J.E. Beasley \& Y.M. Sharaiha. Heuristics for cardinality constrained portfolio optimisation. Computers \& Operations Research, 27(13) (2000), 1271-1302.

[7] S.C. Chiam, K.C. Tan \& A. Al Mamum. Evolutionary multi-objective portfolio optimization in practical context. International Journal of Automation and Computing, 5(1) (2008), 67-80.

[8] K. Deb. Multi-objective optimization using evolutionary algorithms. John Wiley \& Sons (2001).

[9] R.M. Escudero, R.R. Torrubiano \& A. Suárez. Selection of optimal investment portfolios with cardinality constraints, em "IEEE Congress on Evolutionary Computation", pp. 2382-2388, Vancouver, Canadá (2006).

[10] P. Krokhmal, J. Palmquist \& S. Uryasev. Portfolio optimization with conditional value-at-risk objective and constraints. Journal of Risk, 4 (2002), 43-68.

[11] H. Markowitz. Portfolio selection. Journal of Finance, 7 (1952), 77-91.

[12] N.J. Radcliffe. Genetic set recombination. Foundations of Genetic Algorithms, 2 (1992), 203-220.

[13] R.T. Rockafellar \& S. Uryasev. Optimization of conditional value-at-risk. Journal of Risk, 2 (2000), $21-42$.

[14] R.T. Rockafellar \& S. Uryasev. Conditional value-at-risk for general loss distributions. Journal of Banking \& Finance, 26(7) (2002), 1443-1471.

[15] D. Roman, K. Darby-Dowman \& G. Mitra. Mean-risk models using two risk measures: a multiobjective approach. Quantitative Finance, 7(4) (2007), 443-458.

[16] B. Sawik. Downside risk approach for multi-objective portfolio optimization, em "Operations Research Proceedings", Springer Berlin Heidelberg, (2012), 191-196.

[17] H. Soleimani, H.R. Golmakani \& M.H. Salimi. Markowitz-based portfolio selection with minimum transaction lots, cardinality constraints and regarding sector capitalization using genetic algorithm. Expert Systems with Applications, 36(3) (2009), 5058-5063.

[18] R.R. Torrubiano \& A. Suárez. A memetic algorithm for cardinality-constrained portfolio optimization with transaction costs. Applied Soft Computing, 36 (2015), 125-142.

[19] E. Zitzler \& S. Künzli. Indicator-based selection in multiobjective search, em "International Conference on Parallel Problem Solving from Nature”, Springer, (2004), 832-842.

[20] E. Zitzler, M. Laumanns \& L. Thiele et al. Spea2: Improving the strength pareto evolutionary algorithm. Eurogen, 3242(103) (2001), 95-100. 